\title{
Bidirectional fluxes of spermine across the mitochondrial membrane
}

\author{
Silvia Grancara • Pamela Martinis • Sabrina Manente • \\ Aida Nelly García-Argáez • Giampiero Tempera • Marcantonio Bragadin • \\ Lisa Dalla Via $\cdot$ Enzo Agostinelli $\cdot$ Antonio Toninello
}

Received: 1 August 2013/Accepted: 4 September 2013

(C) Springer-Verlag Wien 2013

\begin{abstract}
The polyamine spermine is transported into the mitochondrial matrix by an electrophoretic mechanism having as driving force the negative electrical membrane potential $(\Delta \Psi)$. The presence of phosphate increases spermine uptake by reducing $\Delta \mathrm{pH}$ and enhancing $\Delta \Psi$. The transport system is a specific uniporter constituted by a protein channel exhibiting two asymmetric energy barriers with the spermine binding site located in the energy well between the two barriers. Although spermine transport is electrophoretic in origin, its accumulation does not follow the Nernst equation for the presence of an efflux pathway. Spermine efflux may be induced by different agents, such as FCCP, antimycin A and mersalyl, able to completely or partially reduce the $\Delta \Psi$ value and, consequently, suppress or weaken the force necessary to maintain spermine in the matrix. However this efflux may also take place in normal conditions when the electrophoretic accumulation of the
\end{abstract}

S. Grancara and P. Martinis contributed equally to this work.

S. Grancara · P. Martinis · A. Toninello $(\bowtie)$

Department of Biomedical Sciences, University of Padua, Viale

U. Bassi 58 B, 35131 Padua, Italy

e-mail: antonio.toninello@unipd.it

S. Manente $\cdot$ M. Bragadin

Department of Molecular Sciences and Nanosystems,

Ca' Foscari University of Venice, Venice, Italy

A. N. García-Argáez · L. Dalla Via

Department of Pharmaceutical Sciences, University of Padova,

Via F. Marzolo, 5, 35131 Padua, Italy

G. Tempera $\cdot$ E. Agostinelli

Istituto Pasteur Fondazione Cenci Bolognetti and Department of Biochemical Sciences “A. Rossi Fanelli”, SAPIENZA

University of Rome and CNR, Institute Biology and Molecular

Pathology, Piazzale A. Moro 5, 00185 Rome, Italy polycationic polyamine induces a sufficient drop in $\Delta \Psi$ able to trigger the efflux pathway. The release of the polyamine is most probably electroneutral in origin and can take place in exchange with protons or in symport with phosphate anion. The activity of both the uptake and efflux pathways induces a continuous cycling of spermine across the mitochondrial membrane, the rate of which may be prominent in imposing the concentrations of spermine in the inner and outer compartment. Thus, this event has a significant role on mitochondrial permeability transition modulation and consequently on the triggering of intrinsic apoptosis.

Keywords Mitochondrial permeability transition .

Spermine - Physiological membrane potential .

Reactive oxygen species $\cdot$ Rat liver mitochondria

$\begin{array}{ll}\text { Abbreviations } \\ \Delta \Psi & \begin{array}{l}\text { Electrical transmembrane potential } \\ \text { FCCP }\end{array} \\ & \begin{array}{l}\text { Carbonyl cyanide- } \text {-trifluoromethoxyphenyl- } \\ \text { hydrazone }\end{array} \\ \text { MPT } & \text { Mitochondrial permeability transition } \\ \text { PAO } & \text { Polyamine oxidase } \\ \text { Pi } & \text { Phosphate } \\ \text { RLM } & \text { Rat liver mitochondria } \\ \text { ROS } & \text { Reactive oxygen species } \\ \text { SMO } & \text { Spermine oxidase } \\ \text { SPM } & \text { Spermine }\end{array}$

\section{Introduction}

Natural polyamines, putrescine, spermidine and spermine, are ubiquitous metabolites present in prokaryotic and eukaryotic cells. Structurally they are aliphatic amines 
existing in protonated forms at physiological $\mathrm{pH}$. Table 1 shows the structure of spermine with its $\mathrm{p} K$ values. Polyamines are known to be required for essential physiological processes such as cell growth and cell differentiation and are also involved in neoplastic proliferation (Toninello et al. 2004; Agostinelli et al. 2010a; Montañez et al. 2007). However, it has also been demonstrated that spermine is able to prevent the phenomenon of mitochondrial permeability transition (MPT), an event linked to the triggering of intrinsic apoptosis, and is also able to restore the mitochondrial bioenergetic functions collapsed by the induction of MPT (Sava et al. 2006). Indeed, in the same paper, it has been demonstrated that the main mechanism involved in the protection of MPT is a scavenging effect against the generation of reactive oxygen species (ROS), responsible together with $\mathrm{Ca}^{2+}$ for MPT (Sava et al. 2006). However, also the electrostatic interactions between the positive charges of spermine with the anionic ones present in the transition pore structures may contribute to the protection. However, it may be pointed out that spermine, in particular types of mitochondria, such as those isolated from brain, does not protect mitochondria by a scavenging effect, as in brain the MPT is not induced by ROS (Grancara et al. 2012). Thus, in this case the above-mentioned electrostatic interaction is proposed.

Consistent amounts of polyamines have been detected in the cytosol as a result of the activity of biosynthetic enzymes pathway and/or the uptake of polyamines. When cells are under conditions requiring increased polyamine levels, it results in the increase of polyamine uptake that can compensate for insufficient intracellular and subcellular levels (Alhonen-Hongisto et al. 1980; Pegg 1988; Farriol et al. 1999).

For many years there has been a general opinion, also supported by inadequate experiments, that mitochondria do not possess a polyamine transporter in their membranes. However, polyamines were detected in the matrix of the heart (Tadolini et al. 1985), liver (Mancon et al. 1990) and brain (Tassani et al. 1995) mitochondria, lacking biosynthetic enzymes, and it may be considered as an evidence of the existence of the spermine transporter.

The aim of this minireview is to reappraise the mechanism of spermine transport in mitochondria in the light of its effects on the MPT induction and as a possible regulator of this phenomenon. This latter proposal is supported by the observation that spermine is able to go out from mitochondria by establishing a bidirectional flux, or better, a continuous cycling across the membrane. This cycling depends on the different rates of influx and efflux and provides the required concentration of the polyamine in the matrix. Another objective of this review is to evidence the bioenergetic parameters that can influence the cycling of spermine and the conditions that can modulate this.

\section{Spermine transport in the matrix}

The existence of spermine and other polyamine transport in mammalian mitochondria has been demonstrated by several papers since 1985. Most of the experiments were performed on liver (Toninello et al. 1985, 1988a, b, 1991, 1992a, b; Siliprandi et al. 1988; Salvi et al. 2006; Grillo et al. 2007; Agostinelli et al. 2010a; Martinis et al. 2012), but this mechanism was also demonstrated in the heart (Toninello et al. 1990), brain (Tassani et al. 1995; Battaglia et al. 2010, 2012), and kidney (Arndt et al. 2009). The polyamine transporter is specific for the natural polyamines; agmatine has a different specific transporter, since all other amines or amino acids do not utilize these systems. The structure of spermine transporter, at present, has not yet been identified. However, on the basis of the Nernst-Plank theory (Anderson 1989) and the method developed by Gutfreund (1955), it is possible to state that it is formed by a protein channel, exhibiting two asymmetrical energy barriers, with the energy well that is the site of spermine binding located near the external surface at $1 / 8$ of the length of the channel. The highest barrier corresponding to the rate-limiting constant is located at $1 / 4$ of the length of the channel and has an activation energy of $86 \mathrm{~kJ} /$ mol at physiological membrane potential $(\Delta \Psi)$ (Toninello et al. 2000). Thus, the mechanism of this transport is energy dependent being electrophoretic in origin, operating as an uniport requiring high $\Delta \Psi$ values and exhibiting a non-ohmic flux-voltage relationship (Toninello et al. 1992a).

Table 1 The pKa values of amino groups of spermine (adapted from Frassineti et al. 2003)

\begin{tabular}{|c|c|c|}
\hline & Chemical structure & $\mathrm{p} K \mathrm{a}$ values \\
\hline Spermine & $\mathrm{H}_{2} \mathrm{~N}^{-}$ & $\begin{aligned} \mathrm{p} K \mathrm{a}_{1} & =10.98 \\
\mathrm{p} K \mathrm{a}_{2} & =10.09 \\
\mathrm{p} K \mathrm{a}_{3} & =8.83 \\
\mathrm{p} K \mathrm{a}_{4} & =7.94\end{aligned}$ \\
\hline
\end{tabular}

Net charge of spermine at $\mathrm{pH} 7.4$ is 3.74 , as calculated from these pKa values using the Henderson-Hasselbalch equation 
The energization of the membrane being necessary for spermine transport is provided by the oxidation of $\mathrm{NAD}^{+}$or FAD-dependent dehydrogenases which direct electrons to the respiratory chain. Most of the experiments reported in literature utilized succinate as an energy substrate in the presence of rotenone, since rotenone by inhibiting oxalacetate formation prevents the inhibition of succinate dehydrogenase and permits the maximum uptake of spermine due to the very high imposed $\Delta \Psi$. In the presence of $\mathrm{NAD}^{+}$-dependent substrates, $\Delta \Psi$ is lower and the transport is not so efficient. The results reported in Figs. 1 and 2 show the rate and extent of spermine transport in RLM and the corresponding $\Delta \Psi$ values obtained under different experimental conditions (Toninello et al. 1985, 1988a; Agostinelli et al. 2010a).

As shown in Fig. 1 mitochondrial $\Delta \Psi$ imposed by succinate oxidation plus rotenone has a value of about $160 \mathrm{mV}$ that permits a total uptake of spermine of about $40 \mathrm{nmol} / \mathrm{mg}$ protein in $30 \mathrm{~min}$ of incubation, after an instantaneous binding to the membrane of $12-13 \mathrm{nmol} / \mathrm{mg}$ protein (curves a). In the presence of phosphate in the external medium, $\Delta \Psi$ is raised to $180 \mathrm{mV}$ by the drop in

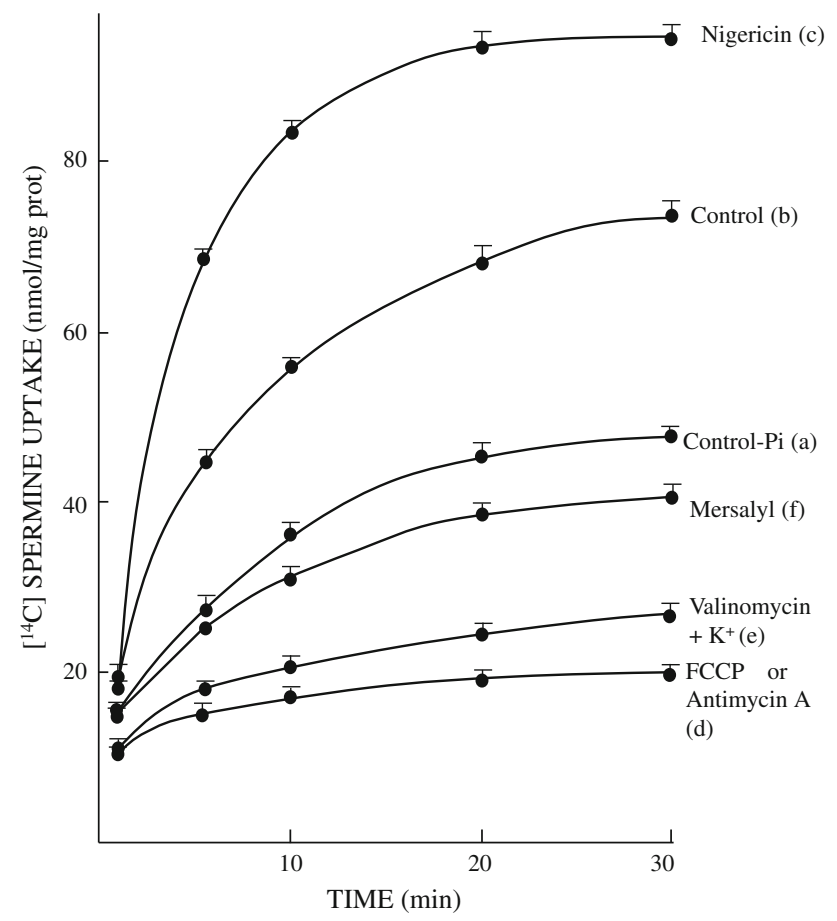

Fig. 1 Spermine transport in different experimental conditions. RLM $\left(1 \mathrm{mg}\right.$ of mitochondrial protein $/ \mathrm{ml}$ ) were incubated at $20^{\circ} \mathrm{C}$ in standard medium [200 mM sucrose, $10 \mathrm{mM}$ Hepes-Cl (pH 7.4), $5 \mathrm{mM}$ succinate, $1.25 \mu \mathrm{M}$ rotenone $]$ containing $1 \mathrm{mM}\left[{ }^{14} \mathrm{C}\right]$ spermine $(50 \mu \mathrm{Ci} / \mathrm{mmol})$. When present, $1 \mathrm{mM} \mathrm{Pi}$, FCCP $(0.1 \mu \mathrm{g} / \mathrm{mg}$ prot $)$, nigericin $(0.33 \mu \mathrm{g} / \mathrm{mg}$ prot $)$, valinomycin $(0.33 \mu \mathrm{g} / \mathrm{mg}$ prot $)$ $+10 \mathrm{mM} \mathrm{KCl}$ (curves $a, b, c, d, e$ ). From: Toninello et al. (1988a). Curve $f$ from Toninello et al. (1985). $\left[{ }^{14} \mathrm{C}\right]$ spermine uptake was determined by centrifugal filtration method described in the above-mentioned references

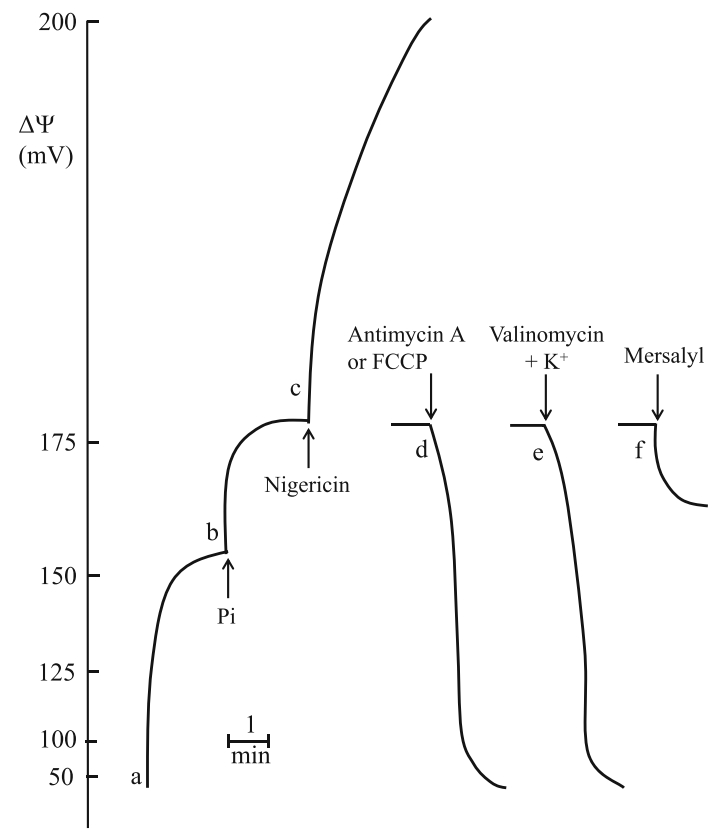

Fig. 2 Mitochondrial $\Delta \Psi$ measurements in different experimental conditions. RLM (1 mg of mitochondrial protein $/ \mathrm{ml}$ ) were incubated in the experimental conditions as in Fig. 1 in the presence of $1 \mathrm{mM}$ $\mathrm{Pi}$. The reported results are in part original (curves $a, b, c$ ) and in part experimental reproductions of previously published results (curves $d$, $e, f)$ from: Toninello et al. $(1985,1988 \mathrm{a}) . \Delta \Psi$ was measured by a tetraphenylphosphonium $\left(\mathrm{TTP}^{+}\right.$) selective electrode (Kamo et al. 1979) corrected using the equation $\Delta \psi=\Delta \psi$ electrode-66.16/0.92 (Jensen et al. 1986). Matrix volume was calculated as $1 \mu \mathrm{l} / \mathrm{mg}$ protein according to Palmieri and Klingenberg (1979). The incubation conditions are the same as reported in the above references

$\Delta \mathrm{pH}$ due to the release of $\mathrm{H}^{+}$subsequent to the entry of phosphate. The mechanism of this effect is explained in the following way: phosphate crosses the membrane as undissociated $\mathrm{H}_{3} \mathrm{PO}_{4}$ and its entry in the matrix is accompanied by dissociation of protons. This leads to a drop in $\Delta \mathrm{pH}$ and consequent increase of $\Delta \Psi$ to about $180 \mathrm{mV}$ due to increased respiration as a physiological answer of mitochondria for maintaining $\Delta \mu_{\mathrm{H}}^{+}$at normal level.

At $180 \mathrm{mV}$, spermine transport is increased to about $70 \mathrm{nmol} / \mathrm{mg}$ protein after the above-mentioned instantaneous binding to the membrane (curves b). When phosphate transport is inhibited by mersalyl, $\Delta \Psi$ is not more increased and spermine transport becomes similar to that of the control (curve f). On the contrary, further increase in $\Delta \Psi$ is observed in the presence of nigericin, an antibiotic inducing an exchange of endogenous $\mathrm{K}^{+}$with exogenous $\mathrm{H}^{+}$that leads to an almost complete collapse of $\Delta \mathrm{pH}$ and a consequent augmentation of $\Delta \Psi$ to the maximum value of $220 \mathrm{mV}$. At this $\Delta \Psi$, the rate of spermine transport was strongly increased and its content became $100 \mathrm{nmol} / \mathrm{mg}$ protein after $30 \mathrm{~min}$ of incubation (curve c). If mitochondria are de-energized by the uncoupler FCCP or the 
respiratory chain inhibitor antimycin $\mathrm{A}$, spermine transport is strongly inhibited, while membrane binding is yet observable although at lesser extent $(\approx 10 \mathrm{nmol} / \mathrm{mg}$ protein) than that in energized conditions.

In the presence of these compounds, $\Delta \Psi$ has a value of about $40 \mathrm{mV}$ that most likely explains the very low spermine uptake (curve d).

A similar result is obtained when mitochondria are incubated in the presence of the antibiotic ionophore valinomycin and $\mathrm{K}^{+}$(curves c). In this case, $\Delta \Psi$ is collapsed to $40 \mathrm{mV}$ while $\Delta \mathrm{pH}$ is raised to about $3 \mathrm{U}$, thus giving a $\Delta \mu_{\mathrm{H}}^{+}$value of $220 \mathrm{mV}$. In other words, under these conditions mitochondria are energized but spermine transport is almost completely blocked. The above described observation clearly demonstrates the $\Delta \Psi$ dependence of spermine transport; indeed, the results reported in Fig. 3 also evidence the opposite changes in the electrical and chemical gradients consequent to this transport to maintain constant $\Delta \mu_{\mathrm{H}}^{+}$. As depicted in Fig. 3 the electrophoretic matrix accumulation of the polycationic polyamine induces a significant gradual drop in $\Delta \Psi$ of about $30 \mathrm{mV}$. If mitochondria remain intact during transport, the organelles operating in such a way and maintaining $\Delta \mu_{\mathrm{H}}^{+}$at the maximum level of about $220 \mathrm{mV}$ have to increase $60 \Delta \mathrm{pH}$ value. This is achieved by augmenting the oxygen consumption (see inset in Fig. 2). The electrophoretic uniporter of spermine operates also with spermidine and putrescine and these polyamines competitively inhibit uptake of each other (Toninello et al. 1992a).

Taking into consideration the electrophoretic nature of spermine transport and analyzing the experimentally obtained concentrations of spermine in the matrix, we would like to focus on some contradictions. According to the Nernst theory, the distribution of spermine across the mitochondrial membrane must be described with the equation:

$\Delta \Psi=\frac{2.3 R T}{z F} \log \frac{\mathrm{SPM}_{\text {in }}}{\mathrm{SPM}_{\text {out }}}$

in which $R, T$ and $F$ are the Nernst parameters, $z$ is the charge of spermine that is 4 .

At $25{ }^{\circ} \mathrm{C} \frac{2.3 R T}{z F}=60 \mathrm{mV}$.

Theoretically in these conditions, with $\Delta \Psi=180 \mathrm{mV}$, the ratio $\frac{\left[\mathrm{SPM}_{\mathrm{in}}\right]}{\left[\mathrm{SPM}_{\mathrm{out}}\right]}=10^{12}$.

However, this ratio is far higher than the obtained experimental values. In fact, using an exogenous spermine concentration of $1 \mathrm{mM}$, the maximum amount of the polyamine accumulated in the matrix is about $100 \mathrm{nmol} /$ mg protein, that is, by considering a matrix volume of $1 \mu \mathrm{l}$ it corresponds to the concentration of $100 \mathrm{mM}$. The ratio $\frac{\left[\mathrm{SPM}_{\mathrm{in}}\right]}{\left[\mathrm{SPM}_{\mathrm{out}}\right]}=10^{2}$.

In conclusion, spermine is transported in mitochondria by an electrophoretic mechanism having $\Delta \psi$ as the driving

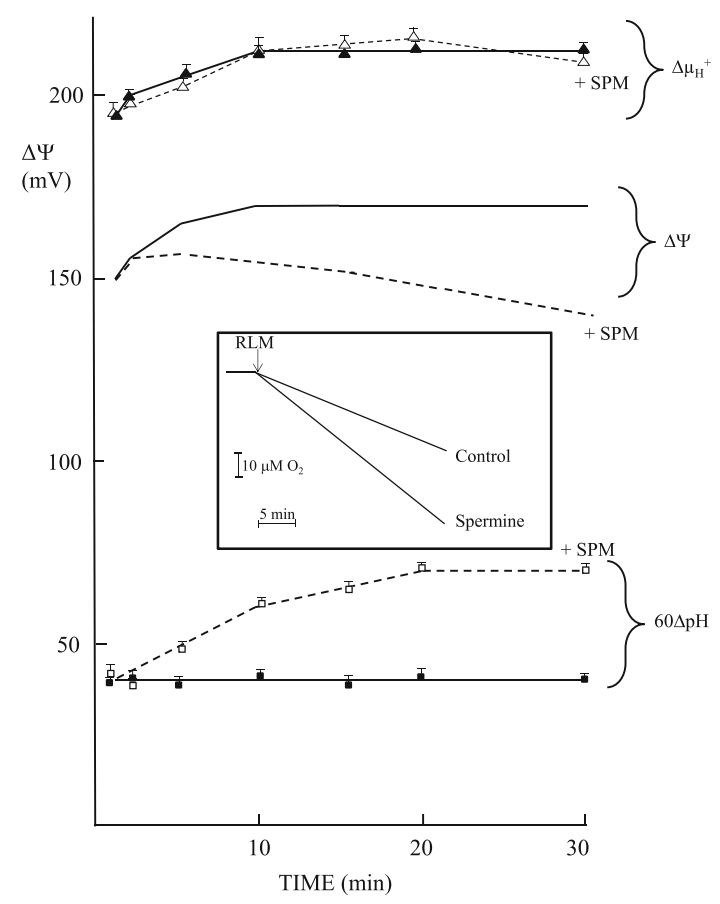

Fig. 3 Changes in $\Delta \Psi$ and $60 \Delta \mathrm{pH}$ during spermine (SPM) transport. RLM ( $1 \mathrm{mg}$ of mitochondrial protein $/ \mathrm{ml}$ ) were incubated in the experimental conditions as in Fig. 1 in the presence of $1 \mathrm{mM} \mathrm{Pi}$. $1 \mathrm{mM}$ spermine was added. $\Delta \Psi$ was measured as in Fig. 2. $\Delta \mathrm{pH}$ was detected by using DMO distribution across the membrane (Rottenberg $1979)$ at the times indicated by the experimental points. $\Delta \mu_{\mathrm{H}+}$ was calculated by the equation $\Delta \mu_{\mathrm{H}+}=60 \Delta \mathrm{pH}+\Delta \Psi$ by extrapolating the ordinate axis and the $\Delta \psi$ values were obtained with the $\mathrm{TPP}^{+}$ electrode at the same times as for $60 \Delta \mathrm{pH}$ determinations. In this regard the $\Delta \psi$ scale has been linearized. In the inset, oxygen uptake was determined by the Clark electrode

force. In fact, phosphate and nigericin by different mechanisms induce an increase in the electrical potential, strongly augmenting spermine uptake. Instead, all the compounds that induce an almost complete or partial drop in $\Delta \psi$ significantly prevent the transport.

\section{Spermine efflux}

The experimentally determined ratio of $\left[\mathrm{SPM}_{\mathrm{in}}\right] /$ $\left[\mathrm{SPM}_{\mathrm{out}}\right]=10^{2}$ and the observation that the electrophoretic accumulation of spermine, instead of completely collapsing $\Delta \Psi$, lower it only slightly, strongly supporting the hypothesis that the polyamine may go out from the organelle after the uptake. Therefore, spermine may resemble somehow the important mitochondria bidirectional transport of $\mathrm{Ca}^{2+}$. This cation is accumulated by an electrophoretic uniport mechanism and is released by an antiport exchange with $\mathrm{Na}^{+}$or $\mathrm{H}^{+}$that is an electroneutral mechanism. Thus, $\mathrm{Ca}^{2+}$ cycles across the mitochondrial membrane with dissipation of energy. On the basis of these 
considerations, further experiments on polyamine transport were performed to demonstrate the presence of an efflux pathway for spermine.

There are several papers showing that spermine may be released from mitochondria, after its accumulation, when the organelles are treated with particular effectors (Toninello et al. 1985, 1988a, b, 1990, 1991; Siliprandi et al. 1988; Tassani et al. 1995; Salvi and Toninello 2003). However, no report analyzes in an in-depth way the mechanism of this process. The results presented in Fig. 4 summarize previous observations together with new unpublished experiments. As depicted, spermine is released after addition of FCCP, antimycin A (Ant A), valinomycin plus $\mathrm{K}^{+}\left(\mathrm{Val}+\mathrm{K}^{+}\right)$and mersalyl (Mers). FCCP is a protonophore that rapidly transports $\mathrm{H}^{+}$into the matrix by inducing an uncoupling effect between oxygen consumption, which is enhanced at a maximum level, and oxidative phosphorylation, which is completely inhibited. Antimycin A blocks electron flux along the respiratory chain at the level of $b_{1}$ complex at the $Q_{n}$ site (Trumpower 1990), and in this case the synthesis of ATP is also completely hampered. On the basis of these characteristics both the compounds induce a complete collapse of $\Delta \mu_{\mathrm{H}}^{+}$with a strong reduction in $\Delta \Psi$ (a residual fixed charge remains) and reverse $\Delta \mathrm{pH}$ (acidic inside). Valinomycin induces an electrophoretic transport of $\mathrm{K}^{+}$against the concentration gradient $\left(\mathrm{K}^{+}\right.$concentration in the matrix is about $150 \mathrm{mM}$ ). On the basis of this effect, $\Delta \Psi$ is almost completely collapsed with a strong increase in $\Delta \mathrm{pH}$, while $\Delta \mu_{\mathrm{H}}^{+}$ remains at normal level. Mersalyl inhibits phosphate transport preventing both the ingress of $\mathrm{H}^{+}$and the increase in $\Delta \Psi$ induced by the anion. This results in a partial drop of $\Delta \Psi$ with a corresponding increase in $\Delta \mathrm{pH}$. The values of the electrochemical gradient and its components under the above-mentioned conditions extrapolated from the results reported in different papers (Toninello et al. 1985, 1992a) are presented in Table 2.

By evaluating the effects of these agents on the above parameters, it is possible to state that the efflux of spermine may be mediated both in energizing and de-energizing conditions, that is with high or completely collapsed $\Delta \mu_{\mathrm{H}}^{+}$.

In de-energizing conditions (presence of FCCP or antimycin $\mathrm{A}$ ), there is a very rapid uptake of $\mathrm{H}^{+}, \Delta \mathrm{pH}$ is reversed (acidic inside) and spermine is maintained completely protonated. Under these conditions spermine is not retained in the matrix by the very low $\Delta \Psi(40$ or $50 \mathrm{mV})$, but goes out driven by its concentration gradient in exchange with $\mathrm{H}^{+}$(external $\mathrm{pH}$ becomes basic). All the other conditions evidence that the efflux of spermine is related to a drop in $\Delta \Psi$ and an increase in $\Delta \mathrm{pH}$. The drop in $\Delta \Psi$ is very strong with valinomycin plus $\mathrm{K}^{+}$as that induced by FCCP and antimycin A, while in the case of mersalyl it is much smaller. The drop in $\Delta \Psi$ is paralleled

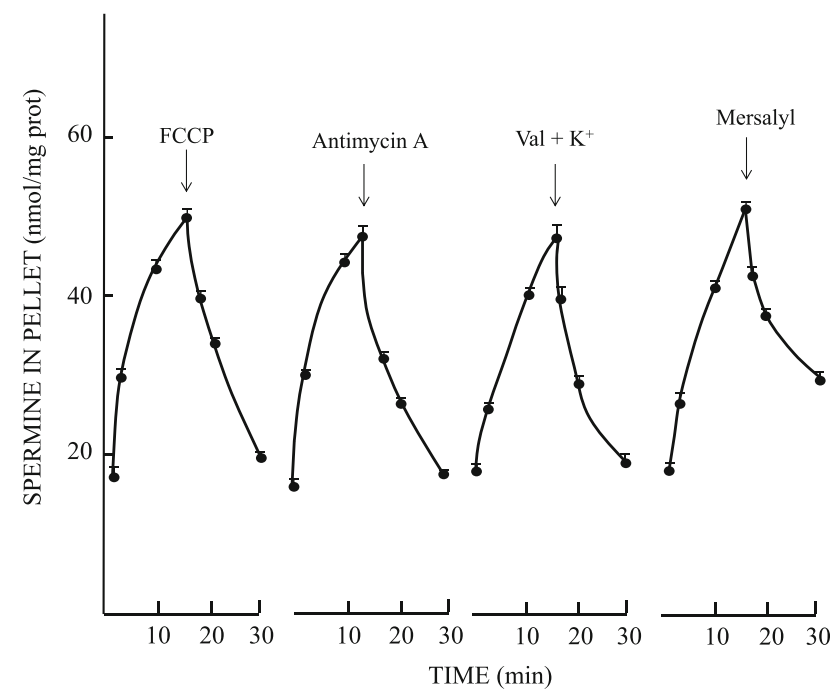

Fig. 4 Spermine efflux induced by drop in $\Delta \Psi$ value. RLM (1 mg of mitochondrial protein $/ \mathrm{ml}$ ) were incubated in the experimental conditions as in Fig. 1 in the presence of $1 \mathrm{mM}$ Pi. When added: FCCP $(0.1 \mu \mathrm{g} / \mathrm{mg}$ prot $)$, antimycin A $(1 \mu \mathrm{g} / \mathrm{mg}$ prot $), 50 \mu \mathrm{M}$ mersalyl. These results are modified experimental reproductions from Toninello et al. (1985). When added valinomycin $(0.33 \mu \mathrm{g} / \mathrm{mg}$ prot $)+10 \mathrm{mM}$ KCl. From: Toninello et al. (1988a). $\left[{ }^{14} \mathrm{C}\right]$ spermine uptake was determined by the centrifugal filtration method described in the above-mentioned references

Table 2 Electrochemical transmembrane potential, $\Delta \mu_{\mathrm{H}}^{+}$and its components, $\Delta \Psi$ and $\Delta \mathrm{pH}$, in energized or de-energized conditions

\begin{tabular}{llclll}
\hline & Control & FCCP & Ant. A & VAL $+\mathrm{K}^{+}$ & MERS \\
\hline$\Delta \mu_{\mathrm{H}}^{+}(\mathrm{mV})$ & 220 & 0 & 0 & 220 & 220 \\
$\Delta \Psi(\mathrm{mV})$ & 180 & 40 & 50 & 60 & 150 \\
$\Delta \mathrm{pH}$ & +0.6 & -1 & -0.83 & +2.65 & +1.15 \\
$60 \Delta \mathrm{pH}(\mathrm{mV})$ & 40 & -40 & -50 & 160 & 70 \\
\hline
\end{tabular}

The sign - indicates acidic $\mathrm{pH}$ in the matrix. The meaning of $60 \Delta \mathrm{pH}$ is explained in the text

by an opposite increase in $\Delta \mathrm{pH}$, to maintain at the maximum level $\Delta \mu_{\mathrm{H}}^{+}$as the energy transducing membranes.

All the above observations of spermine efflux have been obtained in non-physiological conditions-the properties of the inner membrane have been changed by particular effectors. However, the question is: "Is there an experimental evidence demonstrating that spermine may be released from the matrix in conditions near the physiological ones?"

The experimental results presented in Fig. 5 were aimed to answer this question. RLM, incubated in the presence of phosphate and energized by the oxidation of succinate with rotenone in the medium, accumulate $\left[{ }^{14} \mathrm{C}\right]$ spermine for about $30 \mathrm{nmol}$ in $25 \mathrm{~min}$ of incubation (Fig. 5). At that time, the incubation medium containing labeled spermine was substituted with a new one containing cold spermine and phosphate. The incubation was continued for another 
25 min and the amount of $\left[{ }^{14} \mathrm{C}\right]$ spermine present in the mitochondria and also the amount of $\left[{ }^{14} \mathrm{C}\right]$ spermine released in the medium were measured. The amount of labeled spermine in mitochondria diminished first very rapidly and subsequently more slowly. Simultaneously, the amount of labeled spermine present in the medium exhibited a slow, but continuous increase.

The rapid loss of radioactivity in the pellet may be explained by the de-energizing condition induced during the centrifugation of mitochondrial suspension for adding the new medium and also for the release of spermine bound to the membranes. On the contrary, the slower loss of radioactivity after the resuspension of mitochondria in fresh medium containing "cold" spermine is attributed to the release of the labeled spermine out of the matrix. Besides, slow but continuous increase of radioactivity in the supernatant, after the resuspension of mitochondria in the fresh medium, confirms that there is a release of the labeled polyamine during the uptake of "cold" spermine.

The above experiment evidences that the electrophoretic uptake of spermine, in normal energized conditions, is parallel with efflux mechanism, which is most likely electroneutral in origin. The release of spermine takes place after the accumulation of the polyamine has induced a $\Delta \Psi$ drop overcoming a critical threshold. As observed in Fig. 5, after $25 \mathrm{~min}$ of incubation spermine is also released from mitochondria during its uptake. However, after $20 \mathrm{~min}$ there is not any efflux (result not reported). By considering

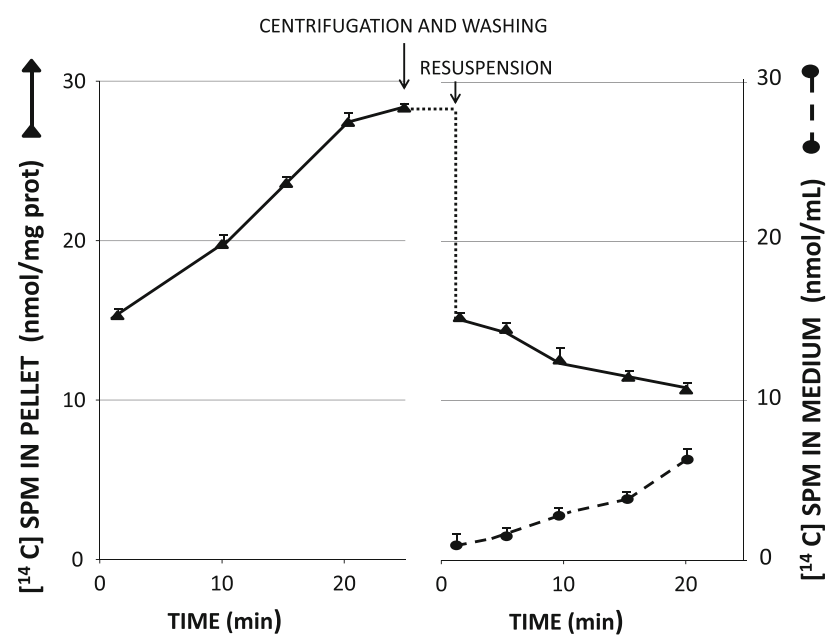

Fig. 5 Spermine cycling across the mitochondrial membrane. Mitochondria were incubated in a standard medium containing $1 \mathrm{mM}$ $\left[{ }^{14} \mathrm{C}\right]$ spermine $(50 \mu \mathrm{Ci} / \mathrm{mmol})$ and $1 \mathrm{mM} \mathrm{Pi}$ (dashed lines with filled triangles). After $25 \mathrm{~min}$ of incubation, mitochondrial suspension was centrifuged at $3{ }^{\circ} \mathrm{C}$ for $2 \mathrm{~min}$ at $173,000 \times g$. The supernatants were discarded, and the pellets were washed three times with cold standard medium and resuspended at $25{ }^{\circ} \mathrm{C}$ in standard medium and incubated for another $20 \mathrm{~min}$ (Toninello et al. 1988a). In addition, after the resuspension, the amount of $\left[{ }^{14} \mathrm{C}\right]$ spermine was also detected in the medium (dashed lines with filled circles) that after 25 or $20 \min \Delta \psi$ has the values of 145 or $150 \mathrm{mV}$, respectively, as assessable in Fig. 3, it is to suggested that spermine efflux begins at $\Delta \psi$ between these values. Thus, when the accumulation of spermine is sufficient to lower $\Delta \Psi$ under this critical threshold value, the mechanism of spermine efflux is triggered by establishing a cycling of the polyamine across the inner membrane. As the mechanism of spermine uptake has been clearly demonstrated to be of electrophoretic origin, the mechanism of spermine efflux is more difficult to explain. One of the common features of the above experiments is that spermine is released when $\Delta \Psi$ is completely or partially collapsed, i.e., when the driving force for the uptake lacks or is weakened. These are the conditions, as mentioned above, obtained by the complete $\Delta \Psi$ collapse by $\mathrm{H}^{+}$re-entry (FCCP, antimycin) or by $\mathrm{K}^{+}$ uptake (valinomycin) that suggest an electroneutral exchange $\mathrm{H}^{+} /$spermine or $\mathrm{K}^{+}$/spermine, respectively.

The effect of mersalyl as mentioned above has to be considered in the light of its effect on phosphate transport. Phosphate is transported bidirectionally by an electroneutral mechanism utilizing $\Delta \mathrm{pH}$ as the driving force. High matrix $\mathrm{pH}$ favors phosphate uptake; high external $\mathrm{pH}$ favors phosphate efflux. Phosphate uptake, in turn, lowers $\Delta \mathrm{pH}$ by favoring $\Delta \Psi$ increase and, consequently, a parallel spermine uptake (Fig. 6). The addition of mersalyl blocks phosphate uptake enhances $\Delta \mathrm{pH}$ (increase of alkaline $\mathrm{pH}$ in matrix) and lowers $\Delta \Psi$ (Table 1). In this condition, spermine goes out accompanied by phosphate (see Fig. 6). If nigericin is added during the parallel efflux of spermine and phosphate, the polyamine is re-accumulated as nigericin induces the highest $\Delta \Psi$ value. Instead, phosphate continues to be released as nigericin also induces a complete drop in $\Delta \mathrm{pH}$. This is a very surprising observation, since in the presence of mersalyl the movements of phosphate should be blocked. However, as previously demonstrated (Toninello et al. 1986), the presence of spermine strongly removes the block by mersalyl and permits the efflux of phosphate either together with spermine or alone, most likely utilizing the transporter (mechanism) different from that involved in uptake. Then the question is: "In what forms do spermine and phosphate exit from mitochondria?" In the presence of the inhibitor matrix, $\mathrm{pH}$ is increased and this diminishes the total positive charge of spermine (this together with the induced drop in $\Delta \Psi$ of about $15 \mathrm{mV}$ (Fig. 2) weakens the force retaining the polyamine in the matrix), thus permitting the efflux of partially deprotonated spermine. Under these conditions phosphate is present in the matrix in the anionic form and cannot electroneutrally go out using its known transporter because of the presence of mersalyl. However, due to the presence of spermine, the mersalyl inhibition is removed and phosphate may exit in the anionic form, using a different transporter and an electrophoretic mechanism. 
Fig. 6 Effect of nigericin on the parallel spermine and phosphate efflux induced by mersalyl. The figure shows an original experiment to improve the study of another one previously published. Experimental conditions and methods are as in Toninello et al. (1985). $1 \mathrm{mM}$ spermine, $1 \mathrm{mM}$ phosphate, $50 \mu \mathrm{M}$ mersalyl and $0.33 \mu \mathrm{g} / \mathrm{mg}$ prot nigericin were present (Toninello et al. 1985)

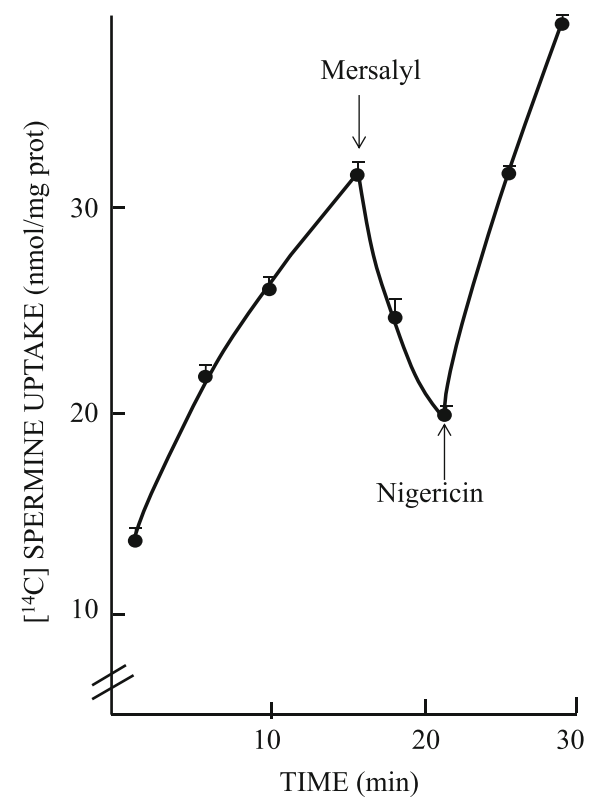

A concluding remark on spermine efflux is that the utilization of the de-energizing agents and the other ones that induce this event evidence by a non-physiological mechanism the existence of an efflux pathway for spermine. However, the release of spermine may take place also in conditions near those physiological, during its electrophoretic uptake, when $\Delta \psi$ is lowered to a critical value. This contemporaneous bidirectional flux of spermine establishes a continuous cycling across the mitochondrial membrane.

\section{Role of spermine cycling}

The observation that spermine can cycle across the inner membrane of energized mitochondria in conditions approximately near to the physiological ones raises the interest of the biological role of this cycling. Until now, discussions regarding the role of polyamines in mitochondria were mainly addressed to discover the effect of their accumulation in the matrix without considering the possible existence of an efflux pathway. As it has been previously reported the uptake of spermine by mitochondria is related to a large numbers of physiological effects. Spermine is able to stabilize mitochondrial membranes; it controls cation fluxes across the membranes, augments the respiratory control ratio and regulates the mitochondrial volume homeostasis (Saris et al. 1969; Chaffee et al. 1977, 1978, 1979; Byczkowski et al. 1982). In this regard, the polyamine modulates $\mathrm{Ca}^{2+}$ transport (Nicchitta and Williamson 1984; Salvi and Toninello 2004) with significant effects at the level of the $\mathrm{Ca}^{2+}$-dependent Krebs cycle intermediates (Wan et al. 1989). In particular, spermine is also able to modulate pyruvate dehydrogenase activity by a mechanism not requiring the presence of $\mathrm{Ca}^{2+}$, but involving the phosphorylation of the subunit $\mathrm{E}_{1 \alpha}$ of pyruvate dehydrogenase (Pezzato et al. 2009). Furthermore, spermine, as mentioned above, favors the transport of phosphate into the matrix and releases the block on this transport caused by mersalyl (Toninello et al. 1986). Again, spermine is able to induce the mitochondrial uptake of several enzymes such as hexokinase (Goncharova et al. 1994), phosphatidate phosphohydrolase (Martin-Sanz et al. 1985), precursor of ornithine carbamoyl transferase (González-Bosch et al. 1991) and in particular CKII and CKI (Bordin et al. 1994; Clari et al. 1994), thus raising the interest to the role of these enzymes in mitochondrial functions. However, a very important effect exhibited by spermine transport in isolated mitochondria is the prevention of $\mathrm{Ca}^{2+}$-dependent induction of the mitochondrial permeability transition (MPT), a phenomenon related to cell death by intrinsic apoptosis. In evaluating the protection of spermine on MPT, it is necessary to consider that in vivo spermine may be oxidized by polyamine oxidase (APAO), spermine oxidase (SMO) and other amine oxidases with the production of aminoaldehydes and hydrogen peroxide (for a review on polyamine oxidation see Agostinelli et al. 2010b). Indeed, preliminary results have shown the presence of SMO in the matrix of liver mitochondria (unpublished results). Thus, the production of reactive oxygen species (ROS) due to the amino oxidases activity makes spermine both a possible inducer of MPT and a protective agent.

All above is regulated by the concentration of spermine present inside and outside mitochondria that can be oxidized. In this regard it is important to take into consideration 
that spermine is also a potent ROS scavenger (Ha et al. 1998; Sava et al. 2006). So, concentration of spermine present in the cytosol and in the mitochondrial matrix has a pivotal role in addressing mitochondria toward MPT induction or protection and, consequently, to induce death or life of the cell.

In this regard the rate between influx and efflux of spermine, i.e., the rate of spermine cycling, assumes a pivotal role in imposing both internal and external concentration of polyamine. Thus, spermine functions may be determined by its concentration, i.e., it can be either a source or a scavenger of ROS. Spermine may also act as inducer or inhibitor of MPT. Therefore, the conditions that influence spermine cycling are important for addressing mitochondria toward the above-mentioned opposite effects.

In conclusion, considering that intrinsic apoptosis, depending on the cell type in which it is induced, may have pathological or physiological significance, one may assume that the induction or protection of the MPT by spermine transport, as well as the modulation of this phenomenon by the rate of spermine cycling, may be significant for cancer, neurodegeneration and other diseases and aging.

Acknowledgments This work was partially supported by the Department of Molecular Sciences and Nanosystems, Ca' Foscari Univesrity of Venice, Italy, "Centrale del Latte" Vicenza, Italy, Italian MIUR (Ministero dell'Istruzione, dell'Università e della Ricerca) (EA), by Istituto Superiore di Sanità "Project Italy-USA" (EA), funds MIUR-PRIN (Cofin) (EA), Fondazione Enrico and Enrica Sovena for the scholarship for Giampiero Tempera and by Istituto Pasteur Fondazione Cenci Bolognetti (EA).

Conflict of interest The authors declare that they have no conflict of interest.

\section{References}

Agostinelli E, Marques MP, Calheiros R, Gil FP, Tempera G, Viceconte N, Battaglia V, Grancara S, Toninello A (2010a) Polyamines: fundamental characters in chemistry and biology. Amino Acids 38:393-403

Agostinelli E, Tempera G, Viceconte N, Saccoccio S, Battaglia V, Grancara S, Toninello A, Stevanato R (2010b) Potential anticancer application of polyamine oxidation products formed by amine oxidase: a new therapeutic approach. Amino Acids 38:353-368

Alhonen-Hongisto L, Seppänen P, Jänne J (1980) Intracellular putrescine and spermidine deprivation induces increased uptake of the natural polyamines and methylglyoxal bis(guanylhydrazone). Biochem J 192:941-945

Anderson OS (1989) Kinetics of ion movement mediated by carriers and channels. Methods Enzymol 171:62-112

Arndt MA, Battaglia V, Parisi E, Lortie MJ, Isome M, Baskerville C, Pizzo DP, Ientile R, Colombatto S, Toninello A, Satriano J (2009) The arginine metabolite agmatine protects mitochondrial function and confers resistance to cellular apoptosis. Am J Physiol Cell Physiol 296:C1411-C1419
Battaglia V, Grancara S, Mancon M, Cravanzola C, Colombatto S, Grillo MA, Tempera G, Agostinelli E, Toninello A (2010) Agmatine transport in brain mitochondria: a different mechanism from that in liver mitochondria. Amino Acids 38:423-430

Battaglia V, Tibaldi E, Grancara S, Zonta F, Brunati AM, Martinis P, Bragadin M, Grillo MA, Tempera G, Agostinelli E, Toninello A (2012) Effect of peroxides on spermine transport in rat brain and liver mitochondria. Amino Acids 42:741-749

Bordin L, Cattapan F, Clari G, Toninello A, Siliprandi N, Moret V (1994) Spermine-mediated casein kinase II-uptake by rat liver mitochondria. Biochim Biophys Acta 1199:266-2670

Byczkowski JZ, Zychlinski L, Porter CW (1982) Inhibition of the bioenergetic functions of isolated rat liver mitochondria by polyamines. Biochem Pharmacol 31:4045-4053

Chaffee RR, Salganicoff L, Arine RM, Rochelle RH, Schultz EL (1977) Polyamine effects on succinate-linked and alphaketoglutarate-linked rat liver mitochondrial respiration. Biochem Biophys Res Commun 77:1009-1016

Chaffee RR, Arine RM, Rochelle RH, Walker CD (1978) Spermidine effects on rat kidney and liver mitochondrial respiration. In: Campbell RA, Morris DR, Bartos D, Daves GD, Bartos F (eds) Advances in polyamine research. Raven Press, New York, pp $123-128$

Chaffee RR, Arine RM, Rochelle RH (1979) The possible role of intracellular polyamines in mitochondrial metabolic regulation. Biochem Biophys Res Commun 86:293-299

Clari G, Toninello A, Bordin L, Cattapan F, Piccinelli-Siliprandi D, Moret V (1994) Spermine effect on the binding of casein kinase I to the rat liver mitochondrial structures. Biochem Biophys Res Commun 205:389-395

Farriol M, Segovia T, Venereo Y, Orta X (1999) Importance of the polyamines: review of the literature. Nutr Hosp 14:101-113

Frassineti C, Alderighi L, Gans P, Sabatini A, Vacca A, Ghelli S (2003) Determination of protonation constants of some fluorinated polyamines by means of $13 \mathrm{C}$ NMR data processed by the new computer program HypNMR2000. Protonation sequence in polyamines. Anal Bioanal Chem 376:1041-1052

Goncharova NIu, Zelenina EV, Avramova LV (1994) Hexokinase isoenzyme II has a segment responsible for specific interaction of the enzyme with mitochondrial membranes. Biokhimiia $59: 826-837$

González-Bosch C, Marcote MJ, Hernández-Yago J (1991) Role of polyamines in the transport in vitro of the precursor of ornithine transcarbamylase. Biochem J 279:815-820

Grancara S, Battaglia V, Martinis P, Viceconte N, Agostinelli E, Toninello A, Deana R (2012) Mitochondrial oxidative stress induced by $\mathrm{Ca}^{2+}$ and monoamines: different behaviour of liver and brain mitochondria in undergoing permeability transition. Amino Acids 42:751-759

Grillo MA, Battaglia V, Colombatto S, Rossi CA, Simonian AR, Salvi M, Khomutov AR, Toninello A (2007) Inhibition of agmatine transport in liver mitochondria by new charge-deficient agmatine analogues. Biochem Soc Trans 35:401-404

Gutfreund H (1955) Steps in the formation and decomposition of some enzyme-substrate complexes. Disc Faraday Soc 20:167-173

Ha HC, Sirisoma NS, Kuppusamy P, Zweier JL, Woster PM, Casero RA Jr (1998) The natural polyamine spermine functions directly as a free radical scavenger. Proc Natl Acad Sci USA 95: 11140-11145

Jensen BD, Gunter KK, Gunter TE (1986) The efficiencies of the component steps of oxidative phosphorylation. II. Experimental determination of the efficiencies in mitochondria and examination of the equivalence of membrane potential and $\mathrm{pH}$ gradient in phosphorylation. Arch Biochem Biophys 248:305-323

Kamo N, Muratsugu M, Hongoh R, Kobatake Y (1979) Membrane potential of mitochondria measured with an electrode sensitive 
to tetraphenyl phosphonium and relationship between proton electrochemical potential and phosphorylation potential in steady state. J Membr Biol 49:105-121

Mancon M, Siliprandi D, Toninello A (1990) On the presence of polyamines in mitochondria. It $\mathbf{J}$ Biochem 39:278-279

Martinis P, Battaglia V, Grancara S, Dalla Via L, Di Noto V, Saccoccio S, Agostinelli E, Bragadin M, Grillo MA, Toninello A (2012) Further characterization of agmatine binding to mitochondrial membranes: involvement of imidazoline I2 receptor. Amino Acids 42:761-768

Martin-Sanz P, Hopewell R, Brindley DN (1985) Spermine promotes the translocation of phosphatidate phosphohydrolase from the cytosol to the microsomal fraction of rat liver and it enhances the effects of oleate in this respect. FEBS Lett 179:262-266

Montañez R, Sánchez-Jiménez F, Aldana-Montes JF, Medina MA (2007) Polyamines: metabolism to systems biology and beyond. Amino Acids 33:283-289

Nicchitta CV, Williamson JR (1984) Spermine. A regulator of mitochondrial calcium cycling. J Biol Chem 259:12978-12983

Palmieri F, Klingenberg M (1979) Direct methods for measuring metabolite transport and distribution in mitochondria. Methods Enzymol 56:279-301

Pegg AE (1988) Polyamine metabolism and its importance in neoplastic growth and a target for chemotherapy. Cancer Res 48:759-774

Pezzato E, Battaglia V, Brunati AM, Agostinelli E, Toninello A (2009) $\mathrm{Ca}^{2+}$-independent effects of spermine on pyruvate dehydrogenase complex activity in energized rat liver mitochondria incubated in the absence of exogenous $\mathrm{Ca}^{2+}$ and $\mathrm{Mg}^{2+}$. Amino Acids 36:449-456

Rottenberg H (1979) The measurement of membrane potential and deltapH in cells, organelles, and vesicles. Methods Enzymol 55:547-569

Salvi M, Toninello A (2003) Reciprocal effects between spermine and $\mathrm{Mg}^{2+}$ on their movements across the mitochondrial membrane. Arch Biochem Biophys 411:262-266

Salvi M, Toninello A (2004) Effects of polyamines on mitochondrial $\mathrm{Ca}^{2+}$ transport. Biochim Biophys Acta 1661:113-124

Salvi M, Battaglia V, Mancon M, Colombatto S, Cravanzola C, Calheiros R, Marques MP, Grillo MA, Toninello A (2006) Agmatine is transported into liver mitochondria by a specific electrophoretic mechanism. Biochem J 396:337-345

Saris NEL, Wikström MKF, Seppälä AJ (1969) The effects of oligoamines on cation binding in mitochondria. In: Ernster L, Drahota Z (eds) Mitochondria-structure and function. Academic Press, New York, pp 363-368

Sava IG, Battaglia V, Rossi CA, Salvi M, Toninello A (2006) Free radical scavenging action of the natural polyamine spermine in rat liver mitochondria. Free Radic Biol Med 41:1272-1281
Siliprandi N, Siliprandi D, Toninello A, Garlid K (1988) Polyamine transport in liver mitochondria. In: Palmieri F, Quagliariello E (eds) Molecular basis of biomembrane transport. Elsevier Science Publishers, Amsterdam, pp 155-162

Tadolini B, Cabrini L, Piccinini G, Davalli PP, Sechi AM (1985) Determination of the polyamine content of rat heart mitochondria by the use of heparin-sepharose. Appl Biochem Biotechnol 11:173-176

Tassani V, Ciman M, Sartorelli L, Toninello A, Siliprandi D (1995) Polyamine content and spermine transport in rat-brain mitochondria. Neurosci Res Comm 16:11-18

Toninello A, Di Lisa F, Siliprandi D, Siliprandi N (1985) Uptake of spermine by rat liver mitochondria and its influence on the transport of phosphate. Biochim Biophys Acta 815:399-404

Toninello A, Di Lisa F, Siliprandi D, Siliprandi N (1986) Action of spermine on phosphate transport in liver mitochondria. Arch Biochem Biophys 245:363-368

Toninello A, Miotto G, Siliprandi D, Siliprandi N, Garlid KD (1988a) On the mechanism of spermine transport in liver mitochondria. J Biol Chem 263:19407-19411

Toninello A, Siliprandi D, Castagnini P, Novello MC, Siliprandi N (1988b) Bidirectional transport of spermine across the inner membrane of liver mitochondria. Adv Exp Med Biol 250: 491-496

Toninello A, Dalla Via L, Testa S, Siliprandi D, Siliprandi N (1990) Transport and action of spermine in rat heart mitochondria. Cardioscience 1:287-294

Toninello A, Siliprandi D, Dalla Via L, Siliprandi N (1991) Electrophoretic spermine transport and its effect on phosphate transport in liver mitochondria. Life Chem Rep 9:223-230

Toninello A, Dalla Via L, Siliprandi D, Garlid KD (1992a) Evidence that spermine, spermidine, and putrescine are transported electrophoretically in mitochondria by a specific polyamine uniporter. J Biol Chem 267:18393-18397

Toninello A, Dalla Via L, Testa S, Siliprandi D (1992b) Electrophoretic polyamine transport in rat liver mitochondria. Amino Acids 2:69-76

Toninello A, Dalla Via L, Stevanato R, Yagisawa S (2000) Kinetics and free energy profiles of spermine transport in liver mitochondria. Biochemistry 39:324-331

Toninello A, Salvi M, Mondovì B (2004) Interaction of biologically active amines with mitochondria and their role in the mitochondrial-mediated pathway of apoptosis. Curr Med Chem 11: 2349-2374

Trumpower BL (1990) The protonmotive Q cycle. Energy transduction by coupling of proton translocation to electron transfer by the cytochrome bc1 complex. J Biol Chem 265:11409-11412

Wan B, LaNoue KF, Cheung JY, Scaduto RC Jr (1989) Regulation of citric acid cycle by calcium. J Biol Chem 264:13430-13439 